\title{
Rhizome trait scaling relationships are modulated by climate and are linked to plant fitness
}

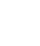

$$
\text { Dinesh Thakur }{ }^{1} \text { and Zuzana Münzbergová }{ }^{1,2}
$$

${ }^{1}$ Institute of Botany, Czech Academy of Sciences, Czech Republic

${ }^{2}$ Department of Botany, Faculty of Science, Charles University, Prague, Czech Republic

Author e-mail: 552.dinesh@gmail.com and zuzmun@natur.cuni.cz

\section{ABSTRACT}

Rhizomes are important organs allowing many clonal plants to persist and reproduce under stressful climates with longer rhizomes indicating enhanced ability of plants to spread vegetatively. However, we do not know how rhizome construction costs (specific rhizome length $\left.\left(\mathrm{SR}_{Z} \mathrm{~L}\right)\right)$ change with increasing length. Here we analysed rhizome length vs mass scaling relationship to address this question. We also analysed plasticity in scaling relationships, its genetic basis, and how scaling relationships are linked to plant fitness. We used data from 275 genotypes of a clonal grass Festuca rubra originating from 11 localities and cultivated under four contrasting climates. Data were analysed using standard major axis regression, mixed effects regression models and structural equation model. We found that rhizome construction costs increase (i.e. lower $\mathrm{SR}_{\mathrm{Z}} \mathrm{L}$ ) with increasing length. The trait scaling relationships were modulated by cultivation climate and its effects also interacted with climate of origin of the experimental plants. Increasing moisture lead to greater increase in rhizome construction costs with increasing length. Our results also demonstrated that trait scaling relationships are linked to plant fitness. This study suggests that scaling relationship are plastic, but also show genetic differentiation and are linked to plant fitness. Therefore, modulation in scaling relationships could be important strategy of plants to persist under variable environments.

Key words: Allometry; Clonal grass; Climate change; Plasticity; Resource allocation 


\section{INTRODUCTION}

Plants optimize allocation of limited resources to different plant parts to increase growth, survival, and reproduction in a given environment (Reich et al. 1997, Wright et al. 2004, Díaz et al. 2016). Changes in environmental conditions may thus modify the allocation patterns via resource trade-offs, which eventually affect allocation to fitness (Springate and Kover 2014, Halbritter et al. 2018). Elucidating the patterns of allocation and determining factors that cause changes in resource allocation patterns is therefore critical for understanding plant carbon economics and hence plant functioning and persistence under current and future climates.

To explore allocation strategies and developing plant economic spectra, trait scaling relationships are very important (Wright et al. 2004, Niklas et al. 2007, Enquist et al. 2015). In last two decades, use of these relationships in ecological research has greatly enhanced our understanding of how resources are allocated into different plant parts such as leaves, twigs or stems (Niklas et al. 2007, Fajardo 2016, Husáková et al. 2018, Deng et al. 2020). The basic concept behind the scaling relationships is that various traits are not independent, but rather are linked to other traits via trade-offs (Reich 2014). The relationship between leaf mass and area is such an example with variable scaling relationships across diverse species and species groups (Milla and Reich 2007, Niklas et al. 2007, Thakur et al. 2019). As a rule in scaling relationships, the value of scaling exponent significantly deviating from 1 indicates that plants disproportionately allocate resources to specific plant functions. For instance, based on value of scaling exponent in the relationship between different plant organs, Smith-Martin et al. (2020) reported differeing allocation patterns to different organs among functional groups (i.e. lianas, evergreen trees, deciduous trees). Klimešová et al. (2017) reported isometric scaling relationship between belowground carbohydrate storage and leaf biomass. Chen et al. (2019) also studied scaling relationships among biomass and production rates of fine root and leaves, and reported that biomass scales isometrically, but production rate of fine roots is less than leaves. Similarly, in case of leaf area and mass, Niklas et al. (2007) inferred that plants allocate disproportionately more resources to form per unit leaf area as leaf size increases (i.e. larger leaves have higher light interception cost).

Trait relationships have been studied widely in last two decades, including studies on aboveground (e.g. Reich et al. 1997, Wright et al. 2004, Niklas et al. 2007, Milla and Reich 2007) as well as belowground traits (e.g. Minden and Kleyer 2014, Poorter et al. 2015, Husáková et al. 2018, Deng et al. 2020). There is also considerable information on how scaling relationships differ across plant functional groups, species, vegetation types and climatic 
regions (Milla and Reich 2007, Niklas et al. 2007, Thakur et al. 2019). Scattered studies reporting changes in stem (in woody species) and root construction costs also exist (Niklas and Enquist 2001, 2002, Chen et al. 2019). However, there is no such information for rhizomes. In this context, recently, Goldberg et al. (2020) highlighted that there is a strong need of addressing questions related to resource allocation to clonal growth in plants.

Rhizomes are ecologically important plant organs responsible for vegetative spread in many plant species (Klimešová and De Bello 2009, Duchoslavová and Jansa 2018), with increased importance in stressful climates e.g. above treeline (Billings and Mooney 1968, Körner 2003). Rhizomes form bud banks (Ott et al. 2019) and enable species persistence by tolerating highly severe conditions (e.g. very low or high temperatures or flooding) and promoting rapid growth during favourable season (because these also act as storage organs) (Billings and Mooney 1968, Hull 2008, Kinmonth-Schultz and Kim 2011). Rhizomes also have important roles in processes such as belowground carbon sequestration (De Deyn et al. 2008), community assembly (Weiher et al. 1998) and plant community resilience to disturbances (Hull 2008, Speed et al. 2010).

Length of the rhizome per unit dry mass investment (i.e. specific rhizome length $\left(\mathrm{SR}_{\mathrm{Z}} \mathrm{L}\right.$ = rhizome length $/$ dry mass)) is a key trait indicative of cost of vegetative spread. Based on patterns reported in literature on aboveground traits, three alternative patterns are expected for scaling relationships between rhizomes length and mass. First, lower construction cost (high $\mathrm{SR}_{z} \mathrm{~L}$ for longer rhizomes) for larger rhizomes which reflect a fast spread strategy using thin or low-density rhizomes with a concomitant high risk of rhizome damage. The other possibility is that construction costs increase (low $\mathrm{SR}_{\mathrm{z}} \mathrm{L}$ for longer rhizomes) with size resulting to highdensity/thick rhizomes that can resist damage. Thirdly, there is no change in $\mathrm{SR}_{z} \mathrm{~L}$ with increasing length. We are, however, not aware of any study differentiating among these three scenarios. Although, there are a few studies that only report differences in specific rhizome length for example under different nutrient availability (Ye et al. 2006) and rhizome burial and breakage (Balestri and Lardicci 2014), we are not aware of any study on scaling relationships between rhizome length and mass.

Climate is a major driver of plant functioning with vast literature on patterns, causes and consequences of functional trait variation along climatic gradients. In contrast, little is known about the effects of climate on trait scaling relationships (Vasseur et al. 2018). Specifically, earlier studies analysing variation in scaling relationships due to climatic factors (e.g. Xiang et al. 2013, Fajardo 2016, Klimešová et al. 2017, Thakur et al. 2019) have not separated the effects of specific environmental factors on these relationships. Also, earlier 
studies have not differentiated between genetic differences and plasticity in scaling relationships. This might be because majority of the earlier studies are carried out in very complex natural environments where multiple factors (e.g. soil properties, climate and genetic changes) act at the same time. We thus need experimental studies allowing to disentangle the role of each factor separately.

In addition to allowing the separation of the effects of single environmental factors, the great advantage of experimental studies is that they allow to assess the specific mechanisms determining the scaling relationships. Specifically, by studying plants from different original conditions cultivated under different environmental conditions, it is possible to assess the relative importance of genetic differentiation and phenotypic plasticity in determining the scaling relationships. Genetic differentiation has a role in generation and maintenance of biodiversity, and therefore allows to get insight into evolutionary processes and the adaptive divergence of populations (Weiher et al. 1998). On the other hand, phenotypic plasticity allows individual organisms to develop appropriate traits that better fit to a particular environment that they encounter (Yan et al. 2013). While studies separating these effects in terms of plant performance are common (e.g. (Münzbergová et al. 2017, Datta et al. 2017, Manzanedo et al. 2019), studies doing so in terms of trait scaling relationships are rare (but see (Vasseur et al. 2018)).

Earlier work on functional traits has linked trait variability with large scale patterns in ecosystem functioning (Diaz et al. 2004, Adler et al. 2014, Funk et al. 2017) and species coexistence in communities (Westoby et al. 2002, McGill et al. 2006). Trait variation has also been linked to plant fitness with specific trait combinations increasing fitness in specific climates (Anderson 2016, Mota et al. 2018). For instance, Kramer-Walter et al. (2016) linked specific root length, Liu et al. (2016) linked specific leaf area and Meyer and Schmid (1999) linked rhizome density to whole plant fitness. However, how changes in trait scaling relationships influence whole-plant fitness, growth and ecosystem function is still unclear (Vasseur et al. 2018). Here, we argue that trait scaling relationships must be linked to plant performance, such as net carbon gain. For example, it can be expected that plants in higher temperature and sufficient moisture would require less protective and supportive tissues, therefore the scaling relationships among traits should indicate no additional cost for larger structures (e.g. leaf area and/or rhizome size). In this case, the saved resources can be allocated to enhance the fitness (e.g. formation of extra structures rather than structures with high cost). This increased investment to fitness is ultimately expected to be reflected in other traits such as photosynthetic rate and total plant biomass. However, there is still dearth of studies that link 
scaling relationships to plant fitness (but see Vasseur et al. 2018) and we are not aware of any such study for rhizome scaling relationships.

To fill the above-mentioned gaps in our knowledge, we aim to understand rhizome length vs mass scaling relationships in general, to test the effect of climate (original and cultivation, representing genetic differentiation and phenotypic plasticity) on these relationships and explore how these relationships are related to plant fitness. Using Festuca rubra as a model plant, we addressed the following questions: (1) How do rhizome length and mass scale with each other? (2) Do rhizome trait relationships change with changes in temperature and moisture of growth conditions and plant origin? (3) Are rhizome trait scaling relationships linked to plant fitness?

We hypothesize that similar to leaf trait scaling relationships, the rhizome length will fail to keep pace with increasing mass. We expect that the scaling relationships will vary with growth conditions, as a result of which the plants growing in warmer and wetter climates should have lower rate of increase in tissue construction costs (because there is no thermal or water limitation). We also hypothesize that the trait relationships differ between plants from different original climate. Additionally, it is expected that lower rhizome construction cost in longer rhizomes would lead to higher plant fitness.

\section{MATERIALS AND METHODS}

\section{Data Set}

The data used in this study are from Münzbergová et al. (2017) on a widespread clonal grass Festuca rubra L. It is distributed in temperate to tundra regions in both hemispheres as well as in tropical mountains (GBIF.org 2021, accessed on 07/03/2021). It reproduces vegetatively by forming both intravaginal and extravaginal tillers on rhizomes but also reproduces by seeds (Münzbergová et al. 2017). While reproducing vegetatively, this species grows varies in the growth form and consequently resource capture strategies creating a continuum of forms between 'phalanx' and 'guerilla strategy (Skálová et al. 1997). Opposite to 'guerella' strategy, the 'phalanx' strategy is characterized by decrease in the length of rhizome internodes and a greater rate of branching (Lopez et al. 1994).

Used dataset represented trait values of 275 genotypes (genotype identification method is given in Šurinová et al. (2019)) collected from 11 localities (25 genotype per locality). These localities are part of a climatic grid (the SeedClim Grid) of factorially crossed temperature and moisture gradients in western Norway (see details in (Klanderud et al. 2015)). All these genotypes were cultivated in 4 growth chambers (Votch 1014), simulating the four climatic 
extremes of the climate grid (cold/warm combined with dry/wet, (with circles in Figure 1)) resulting in 11 localities $\times 25$ genotypes x 4 climates, i.e. 1100 individuals grown in the experiment. Prior to cultivation, the plants were grown in common climate for about 7 months in total (4 months in garden and 3 months in greenhouse) to remove most transgenerational effects (for details see Münzbergová et al. 2017). The experiment was running from end of February to end of August 2015. Because, at the end of experiment, all plants in all the chambers were of similar age, there were no evident differences in belowground plant parts such as maturation stage of rhizomes among different plants in different growth chambers. Some studies suggest that performing experiments in growth chmabers lead to pseudoreplication of the experiment (Hurlbert 1984). This argumentation has been later refuted as non-justified (e.g. Oksanen 2001, Johnson et al. 2016). Most importanly, even Hurlbert (2004) stated that such a setting is not a problem in cases when the main focus is not on the main effects of the chamber climate, but on any interactive effecs, such as the rhizome scaling relationships in our case (see Münzbergová et al. 2017) for extended discussion of the issue).

In mid-June and at the end of August 2015, all the above-ground parts were cut at $3 \mathrm{~cm}$ to simulate mowing in natural conditions. After the final harvest in August, the below-ground parts were carefully sorted into roots and rhizomes. The total length of all rhizomes per plant was measured and all the biomass was then dried to a constant mass at $60{ }^{\circ} \mathrm{C}$ and weighed. The number of rhizomes per plant could not be counted due to their possible braking during their extraction from the soil, so we only have information on their total length. This restrict us from mentioning if the total rhizome length is due to one rhizome or multiple rhizomes. However, this do not have any influence on the results as in any case (i.e. total length is due to one or more rhizomes) the overall longer length of the rhizome(s) per plant would reflect greater ability to spread.

Due to high correlation among rhizome length ( $\mathrm{mm})$ and rhizome mass $(\mathrm{g})$ we analysed their scaling relationships to test if rhizome length and mass increase proportionately (one to one) or disproportionately to each other. In addition, we also used other measured traits from the same individuals (i.e. aboveground biomass $(\mathrm{g})$, number of ramets, total plant biomass $(\mathrm{g})$, net photosynthetic rate (PN) and specific leaf area (SLA, $\left.\mathrm{mm}^{2} / \mathrm{mg}\right)$ ) estimated for the purpose of Münzbergová et al. (2017), Stojanova et al., (2018) and Kosová et al. (2019) to test if plant fitness and performance are linked to changes in the scaling relationships. Among the used traits, aboveground biomass, number of ramets and total plant biomass were used as they are important indicators of plant fitness in this species with predominant clonal reproduction (Münzbergová et al. 2017). Net photosynthetic rate (PN) and specific leaf area (SLA) are key 
above ground traits indicative of plant carbon capture and leaf construction cost. Their relationship with scaling exponent will inform as to how changes in rhizome mass per unit length with increasing length is linked to changes in leaf efficiency. Due to high work load required to measure these two traits, the data on these are only available from 10 genotypes originating from each of the four most extreme climates of the climatic grid: wettest and driest combined with warmest and coldest, i.e. 4 original climates $\times 4$ cultivation climates $\times 10$ genotypes $=160$ measurements in total (same as in Kosová et al. (2019)).

\section{Data analyses}

\section{Standardized major axis regression}

Standardized major axis (SMA) regression as the method commonly used for analysing scaling relationships was used to calculate scaling exponent and elevation ( $\alpha$ and $\beta$, respectively) for the relationship between rhizome length and mass (Niklas et al. 2007, Warton et al. 2012). SMA regression is most appropriate when both variables have errors and there is not a clear distinction between dependent and independent variables (Warton et al. 2012). A large number of earlier studies had used SMA to explore change in dry mass investment with increasing leaf area. It has been described using the formulae: Area $=\beta M a s^{\alpha}{ }^{\alpha}$, where $\beta$ is the elevation and $\alpha$ is the scaling exponent of the log-transformed area vs. mass regression curve (Niklas et al 2007). In case of rhizomes, the formulae will take the form Length $=\beta_{1}$ Mass ${ }^{\alpha}$. Since $S R_{z} L=$ Length/Mass and Mass $=\beta_{2}$ Length $^{\alpha}$, it follows that $S R_{z} L=\left(1 / \beta_{2}\right)$ Length ${ }^{1-\alpha}$. The value of $\alpha>1$ in this equation would indicates that length fails to keep pace with mass (diminishing returns), whereas $\alpha<1$ means the opposite (increasing returns).

Using the SMA regression, first, we estimated scaling exponents and elevation for each cultivation climate across all populations to identify how the general scaling exponent is modulated by cultivation climate. Secondly, we separately estimated the scaling exponents for plants from each original climate (population) grown at each cultivation climate to test if trait scaling relationships of plants from different original climate differ when grown in similar climate. Same analysis will also allow to test if scaling exponents for plants from same climate of origin differ when cultivated in different climates. In all the cases, we tested if the value of scaling exponent is significantly different from 1 using one sample t-test. Using multiple post hoc comparisons, we tested for differences in scaling exponents between plants grown in different cultivation climates and among plants from different original climates grown in different cultivation climates. SMA was done using 'sma()' function with the argument 'multcomp=TRUE' (for multiple comparisons) in the 'smatr' package (Warton et al. 2012) of R Version 4.0.3 (R Core Team and Core R Team 2019). 
Using SMA regression we also explored the effect of direction of climate change on scaling relationships. For this, we estimated change in temperature (ChangeT) and change in moisture (ChangeM) by subtracting cultivation climate values from original climate. For instance, if the original temperature is $6.5^{\circ} \mathrm{C}$ and cultivation temperature is $12.5^{\circ} \mathrm{C}$, the temperature change is $+6^{\circ} \mathrm{C}$ (for more details see Münzbergová et al., (2017)). We then estimated scaling exponents based on differences in moisture or temperature between original and cultivation climate. Because, scaling exponents differed significantly based on changes in moisture, therefore using linear regression (' $\operatorname{lm}()$ ' function in R) we also tested how change in moisture effects scaling exponents (direction of effect).

Mixed effect models

While SMA regression is the best method to analyse the trait relationships, it does not allow to study the effects of different factors and their interactions on these relationships. Also, it does not allow to consider any additional structure of the data (in our case genotypes). We thus also analysed the data using mixed effects regression models (see Husáková et al. (2018) for similar approach). In the model, we used length as dependent variable and mass as explanatory variable with original climate (temperature and precipitation, referred to as Otemp and Omois respectively), cultivation climate (referred to as Ttemp and Tmois) and interactions as fixed factors and genotype as a random factor. Because the model was complex with up to 5 interacting factors, we used the Akaike Information Criterion (AIC) to select the best model (Säfken et al. 2018).

We also used the mixed effects regression to explore the effect of direction of climate change on trait relationships, providing more straightforward interpretation of the possible intreactions between climate of origin and cultivation. For this, we used ChangeT and ChangeM as fixed factors in the model. In the model in this case, effect of change in climate and its interactions with mass were tested. As above, genotype was used as a random factor. Mixed effects regression was done using 'Imer()' function in 'Ime4' package (Bates et al. 2015) of $\mathrm{R}$ Version 4.0.3 (R Core Team and Core R Team 2019). Rhizome length and mass values were log transformed prior to model fitting.

\section{Relationships with fitness}

To get an overview of the effect of the scaling exponent on plant fitness and resource capture traits, we used structural equation modelling (SEM) using the 'sem()' function of the 'lavaan' package (Rosseel 2012) of R. Data points in SEM were values of scaling exponent one for each population and cultivation conditions, and average trait values based on the same 
plants also one for each population and cultivation conditions. In the SEM, we only used the variables that were significantly related to the scaling exponent. The relationship was tested using linear regression (' $1 \mathrm{~m}()$ ' function in R) (for details see supplementary information S1). We considered 3 possible measures of plant fitness in the SEM, number of ramets and aboveground biomass and total biomass. As the scaling exponent was not related to the number of ramets and aboveground biomass, only total biomass was used in the SEM presented. While doing the SEM, the best model was selected based on the lowest AIC value of the model and highest $p$ value of Chi-Square $\left(\chi^{2}\right)$ (Schermelleh-Engel et al. 2003, Barrett 2007). The other measures of goodness of model fit (comparative fit index, Tucker Lewis index, root mean square error of approximation and standardized root mean square residual) as recommended by Coughlan et al. (2008) were also used to determine the model fit. In SEM, we used scaling exponent as the explanatory variable for response variables (rhizome length, SLA, and total biomass). We also used SLA as explanatory variable for PN and rhizome length. PN was also used explanatory variable for rhizome length. SLA and rhizome length were also used as explanatory variables for total biomass (indicator of fitness). Additionally, we also run the model by using biomass excluding rhizomes and total biomass as indicators of fitness, and the results were largely similar (not shown).

\section{RESULTS}

\section{Effects of cultivation climate on the scaling relationships}

Mixed effects regression results revealed that the relationship between rhizome length and mass was affected by both moisture and temperature of the cultivation climate (i.e. driven by phenotypic plasticity), however, the effect of moisture was stronger (Table 1). The effects of temperature and moisture, however, did not interact (Table 1).

In SMA regression, plants cultivated in different cultivation climates differed significantly in the numerical values of $\alpha$, see Table 2 . The value of scaling exponent $(\alpha)$ was significantly greater than 1 in all the four cultivation climates (Table 2 and Figure 2). This indicate that rhizome length generally scales less than one to one with increasing rhizome dry mass. The value of scaling exponent $(\alpha)$ was significantly larger in plants cultivated in wet climates (both warm wet and cold wet) than in dry climates (Table 2 and Figure 2). This indicates that larger rhizomes are even more costly (in terms of biomass investment per unit length) in wet than dry cultivation climates.

Despite significant differences in scaling exponents among the four cultivation climates, value of scaling exponents was always significantly greater than 1 (Figure 2). This 
means that across various cultivation climates, the increases in rhizome mass result in disproportionately less gains in rhizome length (because $\alpha>1$ ) but the gain is even lower in wet climates. When the value of scaling exponents from each climate of origin in each cultivation climate were tested against the value of 1 , it was found that the value of scaling exponent was either equal to 1 or greater than 1 but was never less than 1 . This indicated that there could be proportionate increase in rhizome length and mass or there is disproportionately higher increase in mass than length but length can never increase proportionately greater than mass.

\section{Effects of climate of origin on the scaling relationships}

In the linear mixed effects regression analysis, there were no significant interactions of mass with original climate only (Table 1). The only significant effects of original climate were in interactions with cultivation climate (i.e. Mass:Tmois:Otemp, Mass:Ttemp:Otemp, Mass:Tmois:Ttemp:Otemp in Table 1) indicating that plasticity in the relationships between rhizome length and rhizome mass depends upon the climate of origin. Also, in the SMA results, there were significant differences among plants from different original climates within a cultivation climate (evident only in T3_M1 cultivation climate, (see Table 3 and Figure 3) as well as between cultivation climates (e.g. T1_M1 and T3_M4 in Table 3). Scaling exponents also differed in plants from the same climate of origin when grown in different cultivation climates (e.g. in T2_M1 and T2_M4). The value of scaling exponent for each original climate across all the cultivation climates ranged from 0.903 (statistically not different from 1 (e.g. climate of origin T2_M4 grown in warm and dry (T3_M1) climate)) to 1.395 (significantly greater than 1 (e.g. genotypes from climate T3_M4 grown in cold and wet (T1_M4) climate)).

\section{Effects of climate change on scaling relationships}

In the mixed effects regression analysis based on degree of change in temperature and moisture between climate of origin and cultivation climate, significant interaction between 'Mass' and 'ChangeM' (change in moisture) was found (Table 4). This indicated differing scaling exponents based on change in moisture. SMA regression also revealed similar results and the value of scaling exponent significantly increased with increase in moisture (Figure 4). Change in temperature had no effect on the scaling exponent. This indicates that increasing or decreasing temperatures do not cause any additional change in rhizome construction cost with increasing length, but with increase in moisture the rhizome mass (construction cost) increases disproportionately more than rhizome length at an increased rate.

\section{Relationship between scaling relationships and plant fitness}


High $\mathrm{p}$ value of the $\chi^{2}(0.99)$ as well as other estimated measures (e.g. Tucker Lewis index, root mean square error of approximation etc.) indicated good fit of the structural equation model (supplementary Table S2.5). The model was able to moderately explain the variances in each of the response variables $\left(\mathrm{R}^{2}\right.$ ranged from 0.34 to 0.68$)$ (Figure 5). The model revealed that the increasing value of scaling exponent significantly negatively influenced rhizome length and SLA indicating that both rhizome length and SLA increases with decreasing value of the scaling exponent. The increasing SLA further led to enhanced photosynthetic rate (PN) thereby increasing the total carbon fixed by the plant, but PN was not singficantly related to total biomass. When SLA were higher, total biomass also tend to be higher and was evident in the model from a marginally singficant positive relationship $(\mathrm{p}=0.054)$ between the two. However, increasing SLA also led to decreasing rhizome length. Additionally, longer rhizomes, were also significantly positively linked with higher plant total biomass.

DISCUSSION

Trait scaling relationships have important implications for understanding the plant resource use strategies and ability of plants to acclimate or adapt to variable environmental conditions. In this study, we analysed the rhizome length versus mass scaling relationships and found a considerable variation in the scaling exponents. The main findings emanating from this study are: (i) The rhizome length fails to keep pace with increasing mass, and as a result, with increasing rhizome length the rhizome construction cost increases. (ii) Trait scaling relationships are modulated by climatic conditions in which the plants grow and their effect interacts with climate of origin. (iii) Climate change affects the scaling relationships and wetter climates lead to greater increase in rhizome construction cost with increasing length. (iv) Trait scaling relationships are closely linked to plant performance (e.g. carbon capture capacity by leaves).

\section{Rhizome construction cost increases with increasing length}

Our analyses shown that, scaling relationships for the functional traits that primarily influence plant vegetative spread have a scaling exponent greater than one in all the cultivation climates. Thus, changes in rhizome length fail to keep pace with increasing mass. This indicates that either bulk rhizome-tissue density or thickness (or both) increase as rhizome length increases (causing decreased $\mathrm{SR}_{Z} \mathrm{~L}$ ). The result is similar to those reported for above ground plant traits (e.g. leaf size and leaf mass (Leishman et al. 2007, Price and Enquist 2007, Atkin et al. 2008)). Theoretically, these results means diminishing returns with increase in rhizome 
length (i.e. increasing construction cost for larger rhizomes). This is in parallel with Niklas et al. (2007) who also reported 'diminishing returns' in case of leaves. In case of leaves (Milla and Reich 2007) also reported that leaf construction cost increases with increasing leaf area.

The finding follows the metabolic scaling theory (Enquist et al. 2007) supporting the fact that trait relationships are of similar nature across evolutionary distinct organisms as well as in ecologically distinct functional traits. We assume that this increasing cost could be because rhizomes are also the organs to transport water and minerals to above ground parts, therefore additional mechanical support is required for longer distance transport. Additionally, rhizomes are also storage organs in grasses (for soluble as well as non-soluble carbohydrates (Klimeš et al. 1999, Kinmonth-Schultz and Kim 2011)) but this feature is unlikely to cause higher mass per unit length with increasing length. Although such type of information is missing in literature, we also do not have any reason to expect that storage function of roots would affect scaling between rhizome mass and length (i.e. larger rhizomes should store disproportionately more or less carbohydrates per unit length than smaller ones). Neverthless, testing if rhizome length and stored carbohydrates have isometric relationship is an open question.

Also, the study species is commonly grazed in natural habitats, so longer rhizomes are more vulnerable to herbivore damage via trampling. Therefore, greater investment in mechanical tissues may be helpful in this case as well. In this context, Striker et al. (2006) stated that the porosity of roots in a grass species (Paspalum dilatatum) is lower (density is higher) when exposed to trampling. Although there is additional cost associated with longer rhizomes, longer rhizomes enable positioning of new ramets away from the parents thereby decreasing intra-clone competition and increasing resource availability and the higher investment is thus reasonable. This might be an important strategy of clonal plants and may lead to overall positive returns (e.g. higher dominance in a community).

\section{Scaling relationships are modulated by growth conditions}

Rhizome length vs mass scaling relationships varied among plants cultivated under different climatic conditions demonstrating that scaling relationships are modulated in response to growth conditions. While there are no previous studies on rhizome scaling relationships allowing to compare our results, several previous studies showed the effect of climate on scaling relationships of leaf traits (e.g. Wright et al. 2005; Atkin et al. 2008; Xiang et al. 2013; Thakur et al. 2019). However, all these studies are from natural environments and were not able to separate phenotypic plasticity from genetic differentiation. By using data from experimentally manipulated conditions, we were able to separate multiple effects in this study. 
Our results provided strong evidence to the fact that plant scaling relationships are plastic in nature. This finding is important because evidence of the plasticity in scaling exponents is lacking particularly in plants (see Casasa \& Moczek (2019) for animals). Specifically, these findings indicate the importance of modulation in scaling relationships under variable climates in Festuca rubra. We argue that similar to the role of trait plasticity in plant persistence under variable climates, plasticity in scaling relationships is also important and might be helpful in optimal resource allocation under changing climatic conditions to enhance plant fitness.

The variation in scaling relationships with changing cultivation conditions might help the plant to balance the need for efficient vegetative spread, resource use and protection against desiccation and soil herbivores. However, the direction of the shift in scaling exponent (lower value in drier sites) was un-expected. Majority of the prior literature supports the fact that warmer and wetter conditions favour plant growth (Natali et al. 2012, Buermann et al. 2018) but our data indicate opposite (because higher tissue construction costs have negative consequences). This could be due to wet climate being too wet (simulation of $2700 \mathrm{~mm}$ of annual rainfall) and is not favourable for the species as also claimed by Münzbergová et al., (2017). These too wet conditions may create hypoxic conditions and it is known that plants in hypoxic conditions have thick aerenchymatic belowground structures (Pedersen et al. 2021). To increase thickness there would be more allocation of resources in the tissues and when rhizomes are longer, more thicker tissues can be helpful for longer distance oxygen transport, thereby requiring more allocation. Secondly, a transition towards 'phalanx' resource use strategy (higher number of ramets per unit length) was also evident in this species in wet climates (Supplementary Figure S2.1) and this transition could be responsible for higher rate of increase in rhizome construction cost with increasing length. This can be expected because plants with 'phalanx' strategy have shorter internodes which are linked to increased stem density, and decreased hydraulic conductivity (Jacobsen et al. 2020). Therefore, with increasing length, increased density and maintainance of hydraulic conductivity may impose additional costs. Third, with increasing wetness, there might be increased activity of soil herbivores or parasitic fungi (Velásquez et al. 2018). Hence, additional investments per unit length are needed for protection because tissues with lower carbon content are easier to degrade (Silver and Miya 2001). Overall, our results indicate that it is more economical for target species to form longer rhizomes in conditions with lower water availability and are also prioritized. This was evident in the results as plants in drier conditions had longer rhizomes (Supplementary Figure S2.2). 
The differences in the value of scaling exponents among cultivation climates were apparently very small, but as shown by Milla \& Reich (2007) the small differences in the numerical value of the scaling exponent (among cultivation climates in our case) can translate into very large differences in construction cost per unit length when rhizomes differ greatly in length. These small differences can have huge consequences for the overall plant performance as supported by our results. Interestingly, in this study, the total length of the rhizome in climates with a greater increase in construction cost (e.g. warm and wet) remained far less than in climates with lesser increase in construction cost (see Supplementary Figure S2.2). We argue that there might be economic constraints that limit rhizome elongation (or ability to spread) when construction cost is increasing rapidly. Therefore, this shorter length of the rhizome in warm and wet climates is the negative consequence of greater increase in construction cost. Our result also hints that plants tend to keep the rhizome construction cost under a certain threshold, above which rhizomes are not formed. This was supported by the fact that $\mathrm{SR}_{z} \mathrm{~L}$ was not significantly lower in warm and wet cultivation climate than dry and wet climate but rhizome length was kept short. Moreover, despite shorter rhizomes, the $\mathrm{SR}_{\mathrm{z}} \mathrm{L}$ at the cultivation climates with higher construction costs have higher variance (infered based on trait driver theory (Enquist et al. 2015)) (Supplementary Figure S2.3). This indicated that in such climates, plants are beginning to construct rhizomes with lower construction cost but as a consequence of larger value of scaling exponent, construction cost surpassed to that in drier climates at much shorter lengths.

\section{Genetic differentiation in scaling relationship}

We suspected that scaling relationships might also be result of adaptation to environments at the places of origin. While we did not find any significant interaction between mass and climate of origin, we detected triple interactions between mass, climate of origin and cultivation indicating genetic differentiation in the plasticity of the scaling relationships. This finding provided supporting evidence to possible local adaptation in scaling relationships associated with phenotypic optimization to enhance fitness. This is in line with Vasseur et al. (2018) providing evidence for genetic basis in the scaling relationship of plant dry mass vs growth rate in Arabidopsis thaliana.

In combination with effect of climate change on the scaling relationships, our results support the idea that scaling relationships are modulated significantly by origin as well as cultivation climate at intraspecific levels. Therefore, we suspect that at community level, differences in scaling relationships (reported in many earlier studies on leaf traits (Niklas and

462 Enquist 2001, Milla and Reich 2007, Niklas et al. 2007, Thakur et al. 2019)) could be result of 
both intraspecific (i.e. responses to climate and local adaptation) as well as interspecific effects (i.e. due to species turn over).

\section{Scaling relationships are linked to plant fitness}

The inverse relationship of scaling exponent with total plant biomass indicated that plant fitness is decreased when formation of longer rhizomes becomes more costly (i.e. larger value of scaling exponent). This was also expected because higher investment per unit length can have negative consequences (less investment) for other traits involved in resource acquisition. The SEM results supports that decreased scaling exponent was associated with lower leaf construction cost (i.e. SLA). In turn, this lower leaf construction cost was associated with higher photosynthetic efficiency. Therefore, when leaf construction costs are lower, more light can be intercepted by the same aboveground mass (Milla and Reich 2007) and subsequently more carbon is fixed. Overall, performance of leaves was greatly increased when the scaling exponent was lower.

Our results also indicated that when the value of scaling exponent is lower, longer rhizomes are formed indicating the plants ability to 'vegetatively spread' is also enhanced. Additionally, the net returns as represented by total biomass were also dependent on rhizome length (greater the length higher the returns) (also see supplementary Figure S1.1). The negative relationship between SLA and rhizome length in the SEM indicates that the 'exploitative' resource use strategy of leaves is linked to limited ability of plants to spread vegetatively. The probable reason is that when leaves are more productive, more resources are allocated to roots to exploit more nutrients from soil. This was supported by strong positive relationship between SLA and root biomass (Supplementary figure S2.4).

Our results on the relationship of scaling exponent with leaf traits (PN and SLA) also indicate that changes in below ground tissue construction costs are linked to above ground tissue construction costs. We could have tested this hypothesis in this study, but available data of leaf area and leaf mass did not allow us to do so (as SLA was based on data from leaf fragments). Overall, the findings of this study provided evidence that when scaling relationships are modulated, plant fitness is compromised. The scaling exponents indicating 'diminishing returns' (as defined in Niklas et al. 2007) had negative consequences on plant fitness.

\section{Conclusions}

Our analysis of the scaling of rhizome length vs mass indicates that rhizome length fails to scale one to one with rhizome mass due to which longer rhizome have higher construction 
cost (i.e. lower $\mathrm{SR}_{\mathrm{z}} \mathrm{L}$ ). Our findings provided evidence for plasticity in scaling relationships in plant traits and demonstrated that rate of increase in rhizome construction cost with length depends upon the growth conditions. Among the climatic conditions, moisture of the cultivation conditions was the main determinant of the scaling relationships. Our results also provided additional support for genetic basis in the scaling relationship. Finally, we demonstrated that scaling relationships are closely linked to plant performance and lower scaling exponents (lower increase in construction cost with length) are linked to traits representing acquisitive resource use strategy.

\section{ACKNOWLEDGEMENTS}

We thank POPEKOL discussion group for useful comments on the manuscript. The study was supported by project GACR 19-00522S and partly by institutional research projects RVO 67985939 and MSMT. We thank Vigdis Vandvik and her team to grant access to the SEEDCLIM climate grid where the initial material has been obtained and Verroslava Hadincová for help with the collection of the initial data.

\section{AUTHOR CONTRIBUTIONS:}

ZM owned the original data used in this study. DT and ZM conceived the idea and conceptualized the study. DT analysed the data with help from ZM. DT wrote the draft of the paper with in-depth suggestions, inputs and editing from ZM. Both the authors approved the final version of the manuscript.

\section{CONFLICT OF INTEREST}

The authors declare no conflict of interest

\section{REFERENCES}

Adler, P. B. et al. 2014. Functional traits explain variation in plant life history strategies. Proc. Natl. Acad. Sci. 111: 740-745.

Anderson, J. T. 2016. Plant fitness in a rapidly changing world. - New Phytol. 210: 81-87.

Atkin, O. K. et al. 2008. Using temperature-dependent changes in leaf scaling relationships to quantitatively account for thermal acclimation of respiration in a coupled global climatevegetation model. - Glob. Chang. Biol. 14: 2709-2726.

Balestri, E. and Lardicci, C. 2014. Seagrass response to burial and breakage of expanding horizontal rhizomes: Implications for clone spread. - Mar. Ecol. Prog. Ser. 504: 133145. 
Barrett, P. 2007. Structural equation modelling: Adjudging model fit. - Pers. Individ. Dif. 42: 815-824.

Bates, D. et al. 2015. Fitting Linear Mixed-Effects Models Using lme4. - J. Stat. Softw. in press.

Billings, W. D. and Mooney, H. A. 1968. The Ecology of Arctic and Alpine Plants. - Biol. Rev. 43: 481-529.

Buermann, W. et al. 2018. Widespread seasonal compensation effects of spring warming on northern plant productivity. - Nature 562: 110-114.

Casasa, S. and Moczek, A. P. 2019. Evolution of, and via, Developmental Plasticity: Insights through the Study of Scaling Relationships. - Integr. Comp. Biol. 59: 1346-1355.

Chen, G. et al. 2019. Allometry of fine roots in forest ecosystems (P Thrall, Ed.). - Ecol. Lett. 22: 322-331.

Coughlan, J. et al. 2008. Structural Equation Modeling : Guidelines for Determining Model Fit. - Electron. J. Bus. Res. Methods 6: 53-60.

Datta, A. et al. 2017. Processes affecting altitudinal distribution of invasive Ageratina adenophora in western Himalaya: The role of local adaptation and the importance of different life-cycle stages (S Delzon, Ed.). - PLoS One 12: e0187708.

De Deyn, G. B. et al. 2008. Plant functional traits and soil carbon sequestration in contrasting biomes. - Ecol. Lett. 11: 516-531.

Deng, Q. et al. 2020. Testing allometric scaling relationships in plant roots. - For. Ecosyst. 7: $1-11$.

Diaz, S. et al. 2004. The plant traits that drive ecosystems: Evidence from three continents. J. Veg. Sci. 15: 295-304.

Díaz, S. et al. 2016. The global spectrum of plant form and function. - Nature 529: 167-171.

Duchoslavová, J. and Jansa, J. 2018. The direction of carbon and nitrogen fluxes between ramets in Agrostis stolonifera changes during ontogeny under simulated competition for light. - J. Exp. Bot. 69: 2149-2158.

Enquist, B. J. et al. 2007. Metabolic scaling and the evolutionary dynamics of plant size, form, and diversity: Toward a synthesis of ecology, evolution, and paleontology. - Int. J. Plant Sci. 168: 729-749.

Enquist, B. J. et al. 2015. Scaling from Traits to Ecosystems: Developing a General Trait Driver Theory via Integrating Trait-Based and Metabolic Scaling Theories. - Adv. Ecol. Res. 52: 249-318.

Fajardo, A. 2016. Are trait-scaling relationships invariant across contrasting elevations in the 
widely distributed treeline species Nothofagus pumilio? - Am. J. Bot. 103: 821-829.

Funk, J. L. et al. 2017. Revisiting the Holy Grail: using plant functional traits to understand ecological processes. - Biol. Rev. 92: 1156-1173.

GBIF.org 2021. Festuca rubra L. in GBIF Secretariat (2019). GBIF Backbone Taxonomy. Checklist dataset https://doi.org/10.15468/39omei accessed via GBIF.org on 2021-0307.

Goldberg, D. E. et al. 2020. Allocation to clonal growth: Critical questions and protocols to answer them. - Perspect. Plant Ecol. Evol. Syst. 43: 125511.

Halbritter, A. H. et al. 2018. Trait differentiation and adaptation of plants along elevation gradients. - J. Evol. Biol. 31: 784-800.

Hull, J. C. 2008. Encyclopedia of Ecology. - Encycl. Ecol.: 2818-2824.

Hurlbert, S. H. 1984. Pseudoreplication and the Design of Ecological Field Experiments. Ecol. Monogr. 54: 187-211.

Hurlbert, S. H. 2004. On misinterpretations of pseudoreplication and related matters: A reply to Oksanen. - Oikos 104: 591-597.

Husáková, I. et al. 2018. Species traits and shoot-root biomass allocation in 20 dry-grassland species. - J. Plant Ecol. 11: 273-285.

Jacobsen, A. L. et al. 2020. Node frequency alters stem biomechanics and hydraulics in four deciduous woody species. - J. Wood Sci. 66: 26.

Johnson, S. N. et al. 2016. The importance of testing multiple environmental factors in legume-insect research: Replication, reviewers, and rebuttal. - Front. Plant Sci. 7: 489.

Kinmonth-Schultz, H. and Kim, S. H. 2011. Carbon gain, allocation and storage in rhizomes in response to elevated atmospheric carbon dioxide and nutrient supply in a perennial $\mathrm{C}$ 3 grass, Phalaris arundinacea. - Funct. Plant Biol. 38: 797-807.

Klanderud, K. et al. 2015. The Importance of Biotic vs. Abiotic Drivers of Local Plant Community Composition Along Regional Bioclimatic Gradients (C Armas, Ed.). - PLoS

Klimeš, L. et al. 1999. Carbohydrate storage in rhizomes of Phragmites australis: The effects One 10: e0130205. of altitude and rhizome age. - Aquat. Bot. 64: 105-110.

Klimešová, J. and De Bello, F. 2009. CLO-PLA: the database of clonal and bud bank traits of Central European flora. - J. Veg. Sci. 20: 511-516.

Klimešová, J. et al. 2017. Is the scaling relationship between carbohydrate storage and leaf biomass in meadow plants affected by the disturbance regime? - Ann. Bot. 120: 979985. 
Körner, C. 2003. Alpine Plant Life: Functional Plant Ecology of High Mountain Ecosystems. - Springer Berlin Heidelberg.

Kosová, V. et al. 2019. Ecophysiological traits of a clonal grass in its climate change response. - bioRxiv: 864827.

Kramer-Walter, K. R. et al. 2016. Root traits are multidimensional: specific root length is independent from root tissue density and the plant economic spectrum. - J. Ecol. 104: 1299-1310.

Leishman, M. R. et al. 2007. Leaf trait relationships of native and invasive plants: Community- and global-scale comparisons. - New Phytol. 176: 635-643.

Liu, Y. et al. 2016. Does greater specific leaf area plasticity help plants to maintain a high performance when shaded? - Ann. Bot. 118: 1329-1336.

Lopez, F. et al. 1994. Guerilla vs. Phalanx Strategies of Resource Capture: Growth and Structural Plasticity in the Trunk Trail System of the Harvester Ant Messor barbarus. - J. Anim. Ecol. 63: 127.

Manzanedo, R. D. et al. 2019. A new approach to study local adaptation in long-lived woody species: Virtual transplant experiments (S Ramula, Ed.). - Methods Ecol. Evol. 10: 1761-1772.

McGill, B. J. et al. 2006. Rebuilding community ecology from functional traits. - Trends Ecol. Evol. 21: 178-185.

Meyer, A. H. and Schmid, B. 1999. Experimental demography of rhizome populations of establishing clones of Solidago altissima. - J. Ecol. 87: 42-54.

Milla, R. and Reich, P. B. 2007. The scaling of leaf area and mass: The cost of light interception increases with leaf size. - Proc. R. Soc. B Biol. Sci. 274: 2109-2114.

Minden, V. and Kleyer, M. 2014. Internal and external regulation of plant organ stoichiometry. - Plant Biol. 16: 897-907.

Mota, C. F. et al. 2018. Differentiation in fitness-related traits in response to elevated temperatures between leading and trailing edge populations of marine macrophytes $(\mathrm{G}$ Diaz-Pulido, Ed.). - PLoS One 13: e0203666.

Münzbergová, Z. et al. 2017. Genetic differentiation and plasticity interact along temperature and precipitation gradients to determine plant performance under climate change. - J. Ecol. 105: 1358-1373.

Natali, S. M. et al. 2012. Increased plant productivity in Alaskan tundra as a result of experimental warming of soil and permafrost. - J. Ecol. 100: 488-498.

Niklas, K. J. and Enquist, B. J. 2001. Invariant scaling relationships for interspecific plant 
Niklas, K. J. and Enquist, B. J. 2002. On the vegetative biomass partitioning of seed plant leaves, stems, and roots. - Am. Nat. 159: 482-497.

Niklas, K. J. et al. 2007. "Diminishing returns" in the scaling of functional leaf traits across and within species groups. - Proc. Natl. Acad. Sci. U. S. A. 104: 8891-8896.

Oksanen, L. 2001. Logic of experiments in ecology: Is pseudoreplication a pseudoissue? Oikos 94: 27-38.

Ott, J. P. et al. 2019. The ecology and significance of below-ground bud banks in plants. Ann. Bot. 123: 1099-1118.

Pedersen, O. et al. 2021. Regulation of root adaptive anatomical and morphological traits during low soil oxygen. - New Phytol. 229: 42-49.

Poorter, H. et al. 2015. How does biomass distribution change with size and differ among species? An analysis for 1200 plant species from five continents. - New Phytol. 208: 736-749.

Price, C. A. and Enquist, B. J. 2007. Scaling mass and morphology in leaves: An extension of the wbe model. - Ecology 88: 1132-1141.

R Core Team and Core R Team 2019. R: A language and environment for statistical computing. - R Found. Stat. Comput. Vienna, Austria 2: https://www.R--project.org.

Reich, P. B. 2014. The world-wide "fast-slow" plant economics spectrum: A traits manifesto. - J. Ecol. 102: 275-301.

Reich, P. B. et al. 1997. From tropics to tundra: Global convergence in plant functioning. Proc. Natl. Acad. Sci. 94: 13730-13734.

Rosseel, Y. 2012. lavaan : An R Package for Structural Equation Modeling. - J. Stat. Softw. in press.

Säfken, B. et al. 2018. Conditional model selection in mixed-effects models with cAIC4. $\operatorname{arXiv}$

Schermelleh-Engel, K. et al. 2003. Evaluating the fit of structural equation models: Tests of significance and descriptive goodness-of-fit measures. - MPR-online 8: 23-74.

Silver, W. L. and Miya, R. K. 2001. Global patterns in root decomposition: Comparisons of climate and litter quality effects. - Oecologia 129: 407-419.

Skálová, H. et al. 1997. Within population genetic differentiation in traits affecting clonal growth: Festuca rubra in a mountain grassland. - J. Evol. Biol. 10: 383-406.

Smith-Martin, C. M. et al. 2020. Allometric scaling laws linking biomass and rooting depth vary across ontogeny and functional groups in tropical dry forest lianas and trees. - New 
Speed, J. D. M. et al. 2010. Plant community properties predict vegetation resilience to herbivore disturbance in the Arctic. - J. Ecol. 98: 1002-1013. study on phenological sensitivity and fitness responses to simulated climate warming. Glob. Chang. Biol. 20: 456-465.

Stojanova, B. et al. 2018. Adaptive differentiation of Festuca rubra along a climate gradient revealed by molecular markers and quantitative traits. - PLoS One 13: 1-20.

Šurinová, M. et al. 2019. Temperature and precipitation, but not geographic distance, explain genetic relatedness among populations in the perennial grass Festuca rubra. - J. Plant Ecol. 12: 730-741.

Thakur, D. et al. 2019. Increase in light interception cost and metabolic mass component of leaves are coupled for efficient resource use in the high altitude vegetation. - Oikos 128:

677 254-263.

Vasseur, F. et al. 2018. Adaptive diversification of growth allometry in the plant Arabidopsis thaliana. - Proc. Natl. Acad. Sci. U. S. A. 115: 3416-3421.

Velásquez, A. C. et al. 2018. Plant-Pathogen Warfare under Changing Climate Conditions. Curr. Biol. 28: R619-R634.

Warton, D. I. et al. 2012. smatr 3- an R package for estimation and inference about allometric lines. - Methods Ecol. Evol. 3: 257-259.

Weiher, E. et al. 1998. Community Assembly Rules, Morphological Dispersion, and the Coexistence of Plant Species. - Oikos 81: 309-322.

Westoby, M. et al. 2002. Plant Ecological Strategies: Some Leading Dimensions of Variation Between Species. - Annu. Rev. Ecol. Syst. 33: 125-159.

Wright, I. J. et al. 2004. The worldwide leaf economics spectrum. - Nature 428: 821-827.

Wright, I. J. et al. 2005. Modulation of leaf economic traits and trait relationships by climate. - Glob. Ecol. Biogeogr. 14: 411-421.

Xiang, S. et al. 2013. Contrasting leaf trait scaling relationships in tropical and temperate wet forest species. - Funct. Ecol. 27: 522-534.

Yan, E. R. et al. 2013. Scaling relationships among twig size, leaf size and leafing intensity in a successional series of subtropical forests. - Tree Physiol. 33: 609-617. secalinus under different nutrient supplies. - Ann. Bot. 98: 187-191. 
699

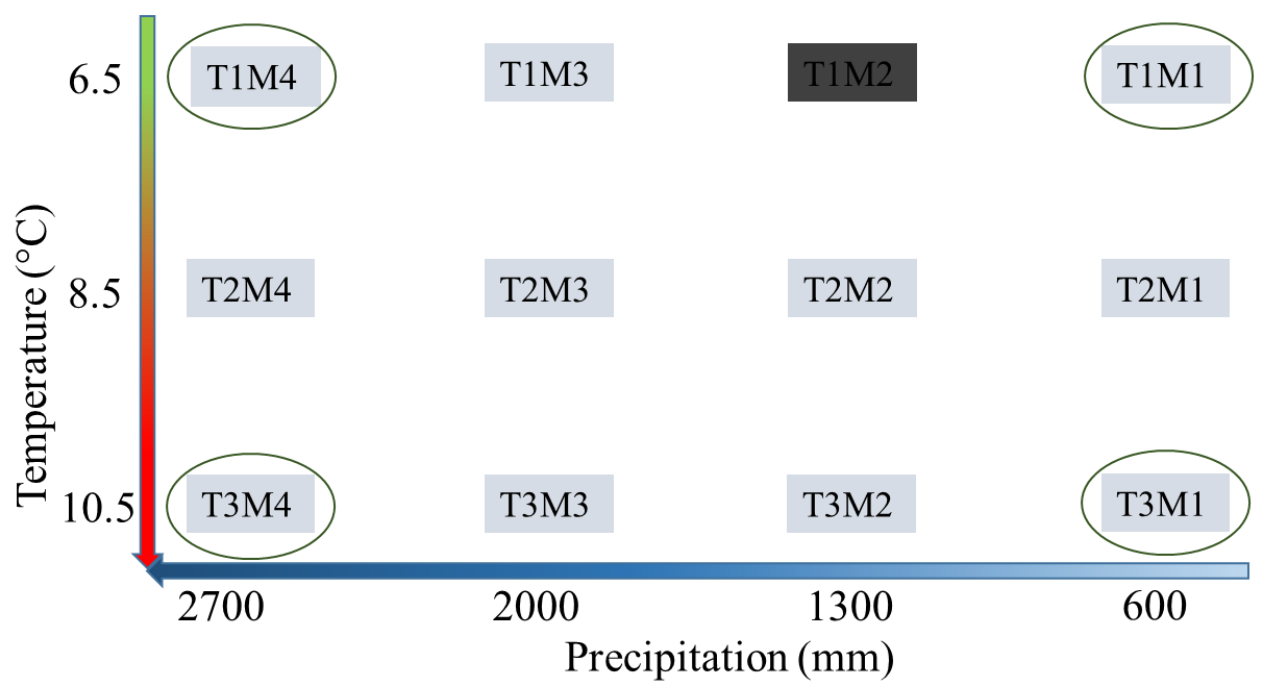

701 Figure 1: Representation of the climatic conditions of the localities from where the plant material was sampled. The four climates that are encircled were also used in climatic chambers as cultivation climates. The climatic condition in dark grey background (T1M2) was not used due to unavailability of the species. 
Table 1. Linear mixed effects regression model describing the effect of rhizome mass $(\mathrm{g})$, original $(\mathrm{O})$ and cultivation $(\mathrm{T})$ climate (temperature and moisture) and their interactions on rhizome length (mm). Genotype was used as a random factor in the model. Only effects of mass alone and in interaction with the other factors are shown here. Full results of the model are given in supplementary Table S2.1. Significant values $(p<0.05)$ are shown in bold.

\begin{tabular}{|l|c|c|}
\hline Fixed factors & F value & P value \\
\hline Mass & $\mathbf{3 0 . 6 7}$ & $<\mathbf{0 . 0 0 1}$ \\
\hline Mass:Tmois & $\mathbf{9 . 3 7}$ & $\mathbf{0 . 0 0 2}$ \\
\hline Mass:Ttemp & $\mathbf{4 . 6 6}$ & $\mathbf{0 . 0 3 1}$ \\
\hline Mass:Omois & 1.17 & 0.279 \\
\hline Mass:Otemp & 2.53 & 0.112 \\
\hline Mass:Tmois:Ttemp & $\mathbf{4 . 6 5}$ & $\mathbf{0 . 0 3 1}$ \\
\hline Mass:Tmois:Omois & 2.80 & 0.095 \\
\hline Mass:Tmois:Otemp & $\mathbf{8 . 1 2}$ & $\mathbf{0 . 0 0 4}$ \\
\hline Mass:Ttemp:Omois & 3.29 & 0.070 \\
\hline Mass:Ttemp:Otemp & $\mathbf{5 . 0 3}$ & $\mathbf{0 . 0 2 5}$ \\
\hline Mass:Omois:Otemp & 0.42 & 0.518 \\
\hline Mass:Tmois:Ttemp:Omois & 1.55 & 0.213 \\
\hline Mass:Tmois:Ttemp:Otemp & $\mathbf{6 . 4 5}$ & $\mathbf{0 . 0 1 1}$ \\
\hline Mass:Tmois:Omois:Otemp & 3.39 & 0.066 \\
\hline Mass:Ttemp:Omois:Otemp & 1.17 & 0.280 \\
\hline
\end{tabular}


713 Table 2. Scaling exponent $(\alpha)$ and elevation $(\beta)$ for scaling relationship between rhizome

714 length ( $\mathrm{mm}$ ) and rhizome mass ( $\mathrm{g}$ ) of Festuca rubra. The plants in different cultivation

715 climates differ among each other in their scaling relationships. Value of scaling exponents in

716 all the cases (presented in bold) were significantly greater than $1.95 \%$ confidence intervals of

$717 \alpha$ and $\beta$ are given in supplementary Table S2.2. The superscript letters represent significant

718 differences in scaling exponent among cultivation climates.

\begin{tabular}{|l|c|c|c|}
\hline Cultivation climate & $\begin{array}{l}\text { Scaling exponent } \\
(\alpha)\end{array}$ & Elevation $(\beta)$ & $\mathrm{R}^{2}$ \\
\hline Cold_Dry & $\mathbf{1 . 0 9 9}^{\mathrm{a}}$ & $-2.768^{\mathrm{a}}$ & 0.925 \\
\hline Cold_Wet & $\mathbf{1 . 2 0 3}^{\mathrm{b}}$ & $-2.904^{\mathrm{b}}$ & 0.846 \\
\hline Warm_Dry & $\mathbf{1 . 0 4 2}^{\mathbf{a}}$ & $-2.768^{\mathrm{a}}$ & 0.935 \\
\hline Warm_Wet & $\mathbf{1 . 2 1 7}^{\mathbf{b}}$ & $-2.981^{\mathrm{b}}$ & 0.849 \\
\hline
\end{tabular}




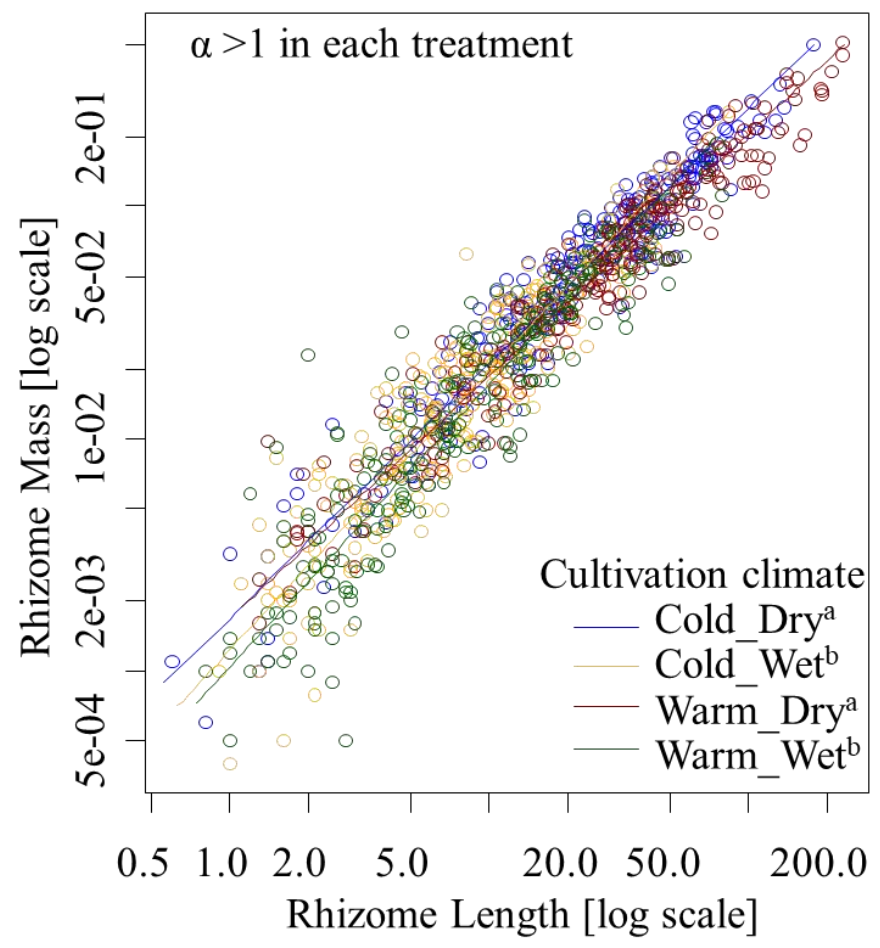

721 Figure 2: Relationship between rhizome length $(\mathrm{mm})$ and mass $(\mathrm{g})$ for plants from different

722 cultivation climates. The superscript letters represent significant differences in scaling 723 exponent among cultivation climates. 
Table 3. Scaling exponent $(\alpha)$ and elevation $(\beta)$ of scaling relationship between rhizome length $(\mathrm{mm})$ and rhizome mass $(\mathrm{g})$ based on origin climate and cultivation climate. In each case the value of $\mathrm{R}^{2}$ was $>0.647$. Value of scaling exponents in bold are significantly greater than $1.95 \%$ confidence intervals of $\alpha$ are given in supplementary Table S2.3. ** represents that scaling exponents differs among original climates for plants grown in the same cultivation climate (in the same column) and ${ }^{\# \#}$ represent that plants from the same original climate differ in scaling exponent among different cultivation climates (in the same row).

\begin{tabular}{|c|c|c|c|c|c|c|c|c|}
\hline & \multicolumn{9}{|c|}{ Cultivation climate } \\
\cline { 2 - 10 } & \multicolumn{2}{|c|}{$\begin{array}{c}\text { Cold_Dry } \\
\text { (T1_M1) }\end{array}$} & \multicolumn{2}{c|}{$\begin{array}{c}\text { Cold_Wet } \\
\left(\mathrm{T} 1 \_M 4\right)\end{array}$} & \multicolumn{2}{c|}{$\begin{array}{c}\text { Warm_Dry } \\
\left(\mathrm{T} 3 \_M 1\right)^{* *}\end{array}$} & \multicolumn{2}{|c|}{$\begin{array}{c}\text { Warm_Wet } \\
\text { (T3_M4) }\end{array}$} \\
\hline $\begin{array}{c}\text { Origin } \\
\text { climate }\end{array}$ & $\boldsymbol{\alpha}$ & $\boldsymbol{\beta}$ & $\boldsymbol{\alpha}$ & $\boldsymbol{\beta}$ & $\boldsymbol{\alpha}$ & $\boldsymbol{\beta}$ & $\boldsymbol{\alpha}$ & $\boldsymbol{\beta}$ \\
\hline T1_M1 & 1.082 & -2.811 & $\mathbf{1 . 3 8 5}$ & -2.993 & $\mathbf{1 . 1 8 3}$ & -2.981 & $\mathbf{1 . 3 1 0}$ & -3.063 \\
\hline T1_M3 & 0.997 & -2.620 & $\mathbf{1 . 2 0 1}$ & -2.906 & $\mathbf{1 . 1 5 6}$ & -2.980 & $\mathbf{1 . 2 6 5}$ & -2.994 \\
\hline T1_M4 & 0.967 & -2.488 & $\mathbf{1 . 2 2 1}$ & -2.952 & 1.005 & -2.622 & 1.115 & -2.861 \\
\hline T2_M1 \#\# & 1.111 & -2.771 & $\mathbf{1 . 2 5 3}$ & -2.884 & 0.982 & -2.652 & $\mathbf{1 . 2 8 1}$ & -2.986 \\
\hline T2_M2 & 1.000 & -2.626 & 1.099 & -2.818 & $\mathbf{1 . 1 7 7}$ & -2.951 & 1.214 & -3.036 \\
\hline T2_M3 & 1.088 & -2.793 & $\mathbf{1 . 2 8 7}$ & -2.988 & 1.034 & -2.774 & $\mathbf{1 . 3 2 4}$ & -3.072 \\
\hline T2_M4 & $\mathbf{1 . 2 9 6}$ & -3.056 & $\mathbf{1 . 2 5 4}$ & -2.988 & 0.903 & -2.557 & $\mathbf{1 . 2 6 6}$ & -3.081 \\
\hline T3_M1 & 1.101 & -2.833 & 1.083 & -2.785 & 0.999 & -2.733 & 1.006 & -2.734 \\
\hline T3_M2 & 1.107 & -2.796 & 1.140 & -2.893 & 0.957 & -2.658 & 1.147 & -2.944 \\
\hline T3_M3 & 1.085 & -2.754 & 1.153 & -2.814 & 1.085 & -2.799 & $\mathbf{1 . 1 9 9}$ & -3.090 \\
\hline T3_M4 & 1.102 & -2.731 & $\mathbf{1 . 3 9 5}$ & -3.064 & 0.986 & -2.660 & 1.066 & -2.647 \\
\hline
\end{tabular}




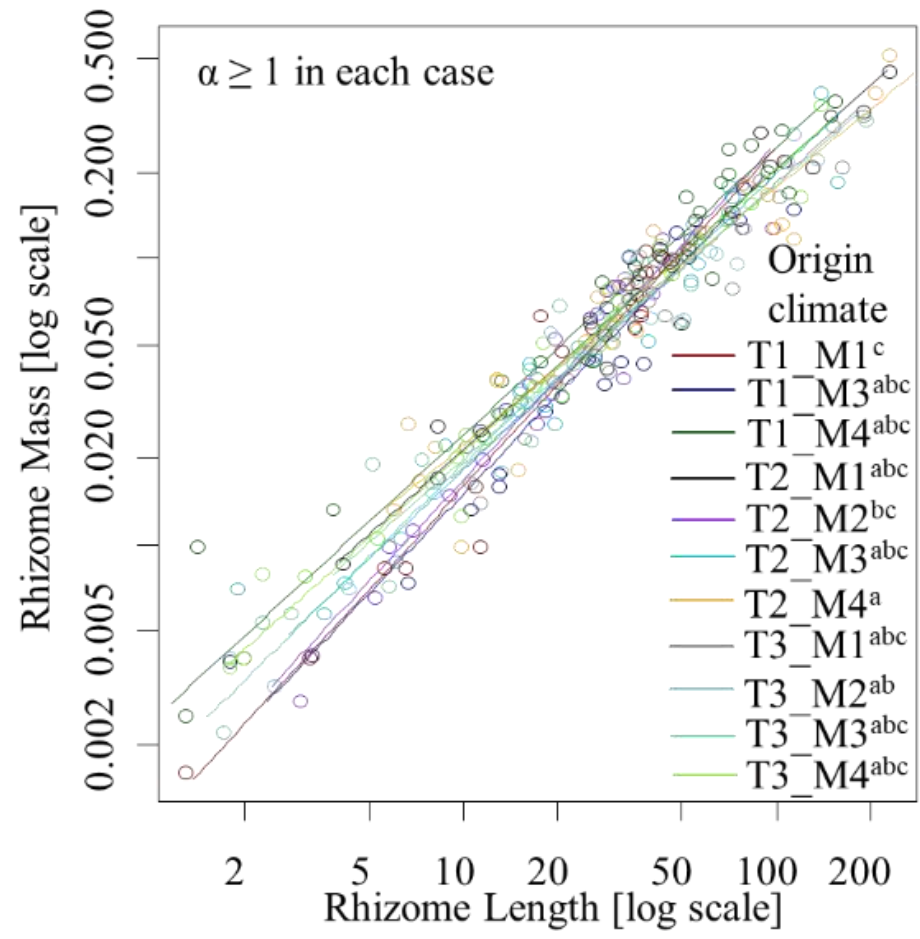

735 Figure 3: Differences in scaling exponent for the relationship between rhizome length (mm)

736 and mass (g) among plants from different original climates grown under warm and dry

737 cultivation climate. The plot is shown only for warm and dry cultivation climate because in

738 other cultivation climates the scaling exponents were statistically not different among each

739 other. T1 to T3 represent origin temperatures from low to high and M1 to M4 represent origin

740 moistures from low to high (for details see Figure 1). 
742 Table 4: Linear mixed effects regression model statistics describing the effect of rhizome mass 743 (g), climate change (temperature and moisture) and their interactions on rhizome length (mm). 744 Genotype was used as a random factor. Only effects of mass alone and its interaction with the 745 other factors are shown here. Full results of the model are given in supplementary Table 2.4. 746 Significant values $(\mathrm{p}<0.05)$ are shown in bold.

\begin{tabular}{|l|c|c|}
\hline Fixed factors & F value & P value \\
\hline Mass & $\mathbf{7 8 5 3}$ & $<\mathbf{0 . 0 0 1}$ \\
\hline Mass:changeT & 2.40 & 0.136 \\
\hline Mass:changeM & $\mathbf{2 0 . 5 1}$ & $<\mathbf{0 . 0 0 1}$ \\
\hline Mass:changeT:changeM & $\mathbf{3 . 8 3}$ & $\mathbf{0 . 0 5 9}$ \\
\hline
\end{tabular}



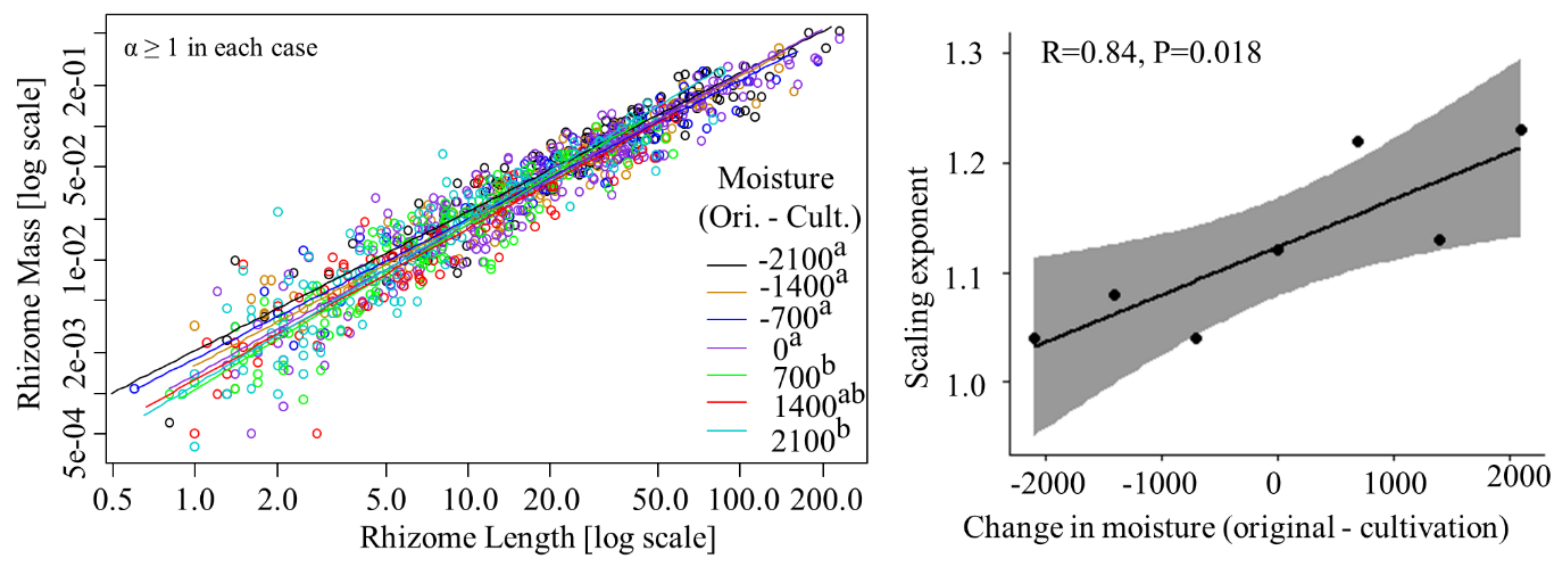

750

$751 \quad$ Figure 4: Figure in the left represents scaling relationship between rhizome length (mm) and 752 mass ( $\mathrm{g}$ ) for plants cultivated under changed moisture $(\mathrm{mm})$ conditions (differences in moisture 753 between origin and cultivation climate). The superscript letters represent significant differences

754 in scaling exponent. The right figure represents the change in scaling exponent with changes 755 in moisture (original - cultivation).

756 


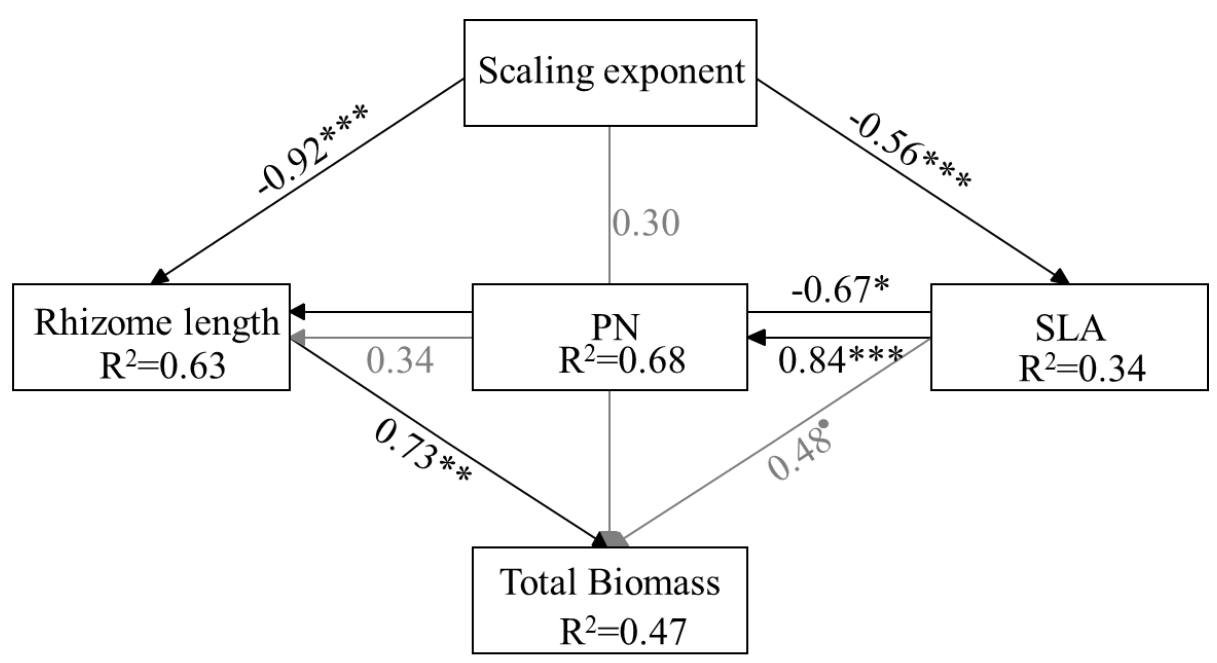

759 Figure 5: Structural equation model representing the effect of scaling exponent on traits related to plant fitness. $\mathrm{PN}=$ net photosynthetic rate, SLA $=$ Specific leaf area $\left(\mathrm{mm}^{2} / \mathrm{mg}\right)$. Path coefficients between variables are unstandardized regression coefficients and part of the variances explained by the model $\left(\mathrm{R}^{2}\right)$ are given under the variable names. The black arrows with regression coefficients in black letters are significant regressions $(\mathrm{p} \leq 0.05)$, while those in grey are non-significant $(\mathrm{p}>0.05)$. $* * *$ indicates $\mathrm{p}<0.001, *$ indicates $\mathrm{p}<0.05$ and $\bullet$ indicates $\mathrm{p}<0.1$. Goodness of fit statistics of the model are given in supplementary Table S2.5. 
bioRxiv preprint doi: https://doi.org/10.1101/2021.05.17.444226; this version posted May 17, 2021. The copyright holder for this preprint (which was not certified by peer review) is the author/funder, who has granted bioRxiv a license to display the preprint in perpetuity. It is made available under aCC-BY 4.0 International license.

768 